\title{
The impact of personality traits on consuming alcohol by genotype: serotonin transporter (5-HTT)
}

\author{
farzaneh zareei, Jaanus Harro \\ University of Tartu /Institute of Psychology, Estonia
}

\begin{abstract}
Researchers have always considered personality traits as an influence on alcohol consumption. This study aimed to investigate the impact of personality traits on alcohol consumption, considering the role of serotonin. This longitudinal study was initiated in 1998, and the study participants $(\mathrm{N}=1176)$ were randomly chosen from 9- and 15-years old schoolchildren from Tartu County in Estonia. The alcohol consumption was divided into two categories of numeric and binary variables. The statistical analyses used in the current study include a simple linear regression, logistic regression, Pearson correlation, independent t-test, and McNamara's test. Results showed that the personality characters, including conscientiousness and agreeableness, were more associated with numeric and binary variables of consuming alcohol than other personality characters regarding the ages of participants. In addition, the mean of neuroticism, agreeableness, and conscientiousness was significantly higher for those who drank alcohol regardless of age. Furthermore, the proportion of alcohol dependence, abuse, was lower now compared to the past. The significant associations between personality traits and consuming alcohol were more common in $\mathrm{L} / \mathrm{L}$ and $\mathrm{S} / \mathrm{L}$ than $\mathrm{S} / \mathrm{S}$ genotype. The current study concludes that neuroticism, agreeableness, and conscientiousness were essential variables that affect alcohol consumption. This relationship between alcohol consumption and personality traits is more common in the $\mathrm{L}$ / L and S / L genotypes.
\end{abstract}

Keywords: 5HTTLPR; Alcohol; Genotype; Personality; Serotonin 